\title{
An Integrated Model for Understanding and Developing Resilience in the Face of Adverse Events
}

\author{
lan de Terte and Julia Becker \\ Joint Centre for Disaster Research, School of Psychology, Massey University, New Zealand
}

\author{
Christine Stephens \\ School of Psychology, Massey University, New Zealand
}

\begin{abstract}
The Five Part Model or Five Areas Model has been a pivotal component of cognitive behavioura therapy and the treatment of various psychological disorders. The Five Part Model consists of five components that represent the interaction of an individual's environment, thoughts, feelings, behaviours, and physical reactions. To date the Five Part Model has primarily been used to understand what has already happened to an individual; for example, when an individual is suffering from posttraumatic stress disorder or is being treated for other psychological difficulties. However, there has been limited application of the Five Part Model to the concept of resilience. Given the effectiveness of the model as an assessment and treatment tool, then it is possible that this model would also be effective in understanding how an individual deals with the aftermath of traumatic events such as disasters. Individual psychological variables are an essential part of whether an individual is resilient to adverse circumstances. However, this model should include as part of the individual's environment wider family, community and societal attributes. It is suggested that the Five Part Model be expanded to include such family, community and societal attributes to account for the influence these have on an individual's psychological resilience. A multidimensional model of resilience is proposed that will need to be scientifically tested; however, it is proposed that this model has application to the variety of disciplines that are involved in the domain of resilience. In addition, this model attempts to address any interdisciplinary barriers that exist, by ensuring that individual, family, community, and societal attributes are considered holistically as part of resilience building.
\end{abstract}

Keywords: Five part model, resilience, social environment

The term 'resilience' has been defined in many different ways, and at a basic level it implies an ability to avoid, overcome, or adapt to adverse circumstances. The widely accepted definition of resilience in the clinical psychology literature is the ability of an individual to bounce back after excessive stress (Agaibi \& Wilson, 2005). However, the term resilience has also been defined in many other ways and used in a variety of contexts, including physical, ecological, social, community, and individual contexts (Norris, Stevens, Pfefferbaum, Wyche, \& Pfefferbaum, 2008). The notion of resilience is appealing, but when considering resilience, many questions arise: What is resilience? How can resilience be achieved? Can resilience be achieved? Should resilience be built before or after a disaster, or during both times? Should the development of resilience be tackled at an individual level or a wider community level? Whose responsibility is it to build resilience? Is it possible to build resilience from a psychological and community perspective?

Different disciplines have approached these questions in different ways. The engineering sciences are primarily interested in resilience of the physical environment. An ecological systems approach is focused on resilience that arises from interactions between important systems (e.g., people and the environment). The health sciences are interested in resilience relating to individual mental and physical wellbeing. Disaster researchers are interested in creating disaster-resilient societies. In our experience many of these disciplines 
operate separately and do not link with parallel, but relevant resilience research. Despite current disciplinary barriers, there is great potential for approaches to resilience to become more multidisciplinary.

In addition to the existence of barriers between disciplines, there are inconsistencies in the scientific investigation of resilience. There is often a strong focus on creating resilient individuals, with little thought given to how individuals interact with wider society and how this influences resilience. Mohaupt (2008) argued that approaches to resilience have often been very individual focused, with an under-representation of research on community influences, social capital, and networks.

We prefer Bonnano's (2004) definition of resilience that postulates that resilience is the ability of an individual to maintain healthy psychological and physical wellbeing despite being exposed to adversity. However, there are limitations to this definition in that it does not include the wider community aspects that appear to influence resilience. To address this discrepancy, we present an integrated Five Part Model that addresses resilience from an individual perspective and incorporates wider community influences. This article will outline psychological resilience, the Five Part Model in its current form, the conceptualisation of resilience with the Five Part Model, a new integrated Five Part Resilience Model, and discuss future directions for this model.

\section{Psychological Resilience}

Research in the field of psychological resilience has predominately conceptualised this concept by investigating disadvantaged children or youth (e.g., Harvey \& Delfrabbo, 2004). Furthermore, theorists have looked at what constitutes the concept of resilience by exploring the dichotomy of the presence of psychopathology versus the absence of psychopathology (e.g., Rutter, 2006). Our main interest is how people are resilient when exposed to a traumatic incident or a series of traumatic incidents, such as natural disasters. The notion of psychological resilience in the face of traumatic exposure has evolved from questions over why some individuals have difficulties when exposed to trauma, and why some individuals experience no difficulties (Agaibi \& Wilson, 2005). To understand how such differences occur, and how the needs of people can be addressed, a structure is required in which individual's experiences of trauma can be framed. We propose that psychological resilience may be fruitfully viewed from the perspective of the Five Part Model.

\section{The Five Part Model}

The Five Part Model or Five Areas Model has been a pivotal component of cognitive-behavioural therapy in the treatment of various psychological disorders (Giarratano, 2004; Williams \& Garland, 2002). Although the Five Part Model has been an essential element of cognitive-behavioural therapy and clinical psychology, there has been limited published research on the scientific merits of the model. For example, a literature search of the terms 'five part', 'five-part', 'five areas', or 'fiveareas' combined with the term 'cognitive therapy' in the literature databases PsycINFO, PsycCRITIQUES, PsycBOOKS, ERIC, Journals@Ovid, International Bibliography of the Social Sciences, Lippincott Williams and Wilkins Journals, The Lancet Archive, MEDLINE, and the Web of Science yielded 43 results. In addition, some of these results did not relate to the Five Part Model conceptualisation portrayed in cognitive-behavioural therapy.

The Five Part Model has evolved as a pragmatic representation of cognitive-behavioural therapy principles (Williams \& Garland, 2002). The model proposes an interaction between an individual's environment, thoughts, feelings, behaviours, and physical reactions. The core component of this model is that individual cognitions affect emotions, physical reactions and in turn, behaviour (Williams \& Garland, 2002). It is generally accepted in the cognitive-behavioural literature that these four components are interrelated (see Beck, 1995). These four components are seen as part of the individual's psychological makeup, which we refer to as 'internal factors.' The individual then operates within a broader social and physical environment (Curran, Machin, \& Gournay, 2006), which we refer to as 'external factors.' The Five Part Model is visually depicted in Figure 1.

\section{The Conceptualisation of Resilience With the Five Part Model}

The Five Part Model allows us to draw diverse approaches to resilience together and to understand resilience as a multidimensional concept made up of personal, social, and physical resources. The key tenet of this proposed model is that resilience is not something an individual has or does not have. Rather, it is something that can be learned and provides scope for intervention.

Kumpfer (1999) has developed a model of resilience that includes the internal aspects of the Five Part Model and some of the external factors of our model. However, Kumpfer's model differs from our conceptualisation of resilience in that Kumpfer's model includes spirituality as an internal resiliency factor, and risk factors and protective factors are included in the environmental component. In addition, we argue that Kumpfer's model includes static and dynamic factors, whereas our model is based on dynamic variables. We postulate that resiliency is an ever-changing process. Furthermore, we suggest that Kumpfer has included some variables in different parts of the model that are not in the correct domain. For example, empathy is included under the behavioural domain rather than the emotion domain.

Lahad (1993) developed a model entitled BASIC-Ph that is intended to help understand how communities cope with adversity. Similar to the Five Part Model, 
Lahad suggested that cognitions, emotions, and physiological response are all important dimensions that have an impact on the way people cope with adverse circumstances. Beliefs, values, and imagination are also identified as distinct considerations in this model, but behaviours are not directly addressed. Social interaction is noted as being important, with the need to link with family and community when dealing with trauma. In contrast to Lahad, the Five Part Model provides a streamlined approach to understand psychological resilience, taking into account the key components of cognitions, emotions, and physical activities, and also accounting for behaviour as a distinct consideration. The following sections outline the key internal and external components that make up the Five Part Model.

\section{Internal Factors Cognitions}

In the Five Part Model, the cognitive aspect of the model refers to how we think or interpret a situation (Williams \& Garland, 2002). If a person is anxious or depressed they often have what is referred to as cognitive distortions or unhelpful thinking styles (Beck, 1995; Leahy \& Holland, 2000). The conceptualisation of the cognitive component of the Five Part Model is clinically useful when treating an individual with depression or anxiety. However, these unhelpful thinking styles may only present themselves when an individual is suffering from depression or anxiety. From a treatment perspective and an intervention perspective it would be useful to understand how people who are resilient think. Therefore, considering the scientific evidence it appears that essential cognitive components of resilience are an individual's optimism, problem-solving skills, perseverance, and resourcefulness (Polk, 1997). These factors have been shown to protect individuals in times of adversity. For example, Chang (2002) illustrated how higher levels of optimism were associated with lower psychological difficulties and greater life satisfaction.

\section{Emotions}

In the Five Part Model, the emotion component of the model refers to an individual's feelings or emotional experiences. As previously mentioned this model is interactive and an individual's feelings may be caused by their unhelpful thinking style or their low mood may cause their unhelpful thoughts. For example, an individual who is feeling low may be sleeping badly, may be staying in bed longer, and may be thinking they are a 'bad person' (Williams \& Garland, 2002). In relation to the conceptualisation of resilience in the framework of the Five Part Model, again it would be difficult to ascertain an individual's altered feelings until they seek psychological input. From a review of the literature on resilience the emotional component of the model appears to be a difficult aspect to determine.
Nevertheless, given the important aspect of this 'cog in the wheel' it is argued that an individual's emotional competence is the key component in the Five Part Resilience Model. However, a shortcoming with emotional competence is how to measure or assess it and it is argued that emotional competence is closely related to emotional intelligence. In this model, although the five components are visualised as equitable factors, it is proposed that emotional competence is a key component to this model because if an individual is not emotionally component or has emotional difficulties, then mental health issues may arise. In addition, emotional intelligence has been identified as a coping mechanism (e.g., Montes-Berges \& Augusto, 2007) and as a predictor of traumatic stress (Hunt \& Evans, 2004).

\section{Behaviours}

In the Five Part Model, the behaviour aspect of the model refers to actions individuals may do as a result of one of the other domains of the model. For example, a person who is feeling sad may decide to undertake certain behaviours to try and lift them out of feeling sad (Curran et al., 2006). The behaviour an individual utilises may be helpful or unhelpful. For instance, someone who is feeling stressed may decide to go on a spending spree or go on a drinking binge. These behaviours would be considered to be unhelpful. Alternatively an individual who is feeling sad may choose to do some physical exercise that would be considered a helpful behaviour. Buckley and colleagues (2004) has demonstrated that individuals with mental health consequences due to traumatic events were less likely to engage in adaptive health practices and consequently were at higher risk of medical complications.

\section{Physical Activities}

In the Five Part Model, the physical aspect of the model refers to the physical sensations an individual may experience (Curran et al., 2006). For example, an individual experiencing anxiety may have heart palpations, unexplained perspiration, nausea, muscle tightness, and shortness of breath. However, in relation to resilience, the physical component refers to physical activities an individual does as a preventive measure. This aspect of the model considers matters such as physical exercise, relaxation, rest, and sleep. This component of the model is closely related to the behaviour aspect of the model because it is argued that when the physical component is conceptualised in this way it is difficult to separate out from an individual's behaviour. The physical and behavioural component of this model could be referred to as an individual's health practices.

\section{External Factors}

Environment

In relation to the Five Part Model, the external aspect of the model refers to the environment component. The 
environment refers to a person's external factors (Giarratano, 2004). In the Five Part Model examples of an individual's environment may include their current stressors, their interpersonal relationships, and their previous psychological distress. This model of resilience is conceptualised from an intervention perspective. From a psychological perspective the most relevant environmental feature of the model is social support. Social support has been investigated from the perspective of a protective factor in people who have been exposed to traumatic events (Hyman, 2004; Stephens, 1997) and from the perspective of an individual's network of professional and personal relationships. These networks are important to the model proposed in this article. However, we argue that the environmental aspect of this model is far wider and encompasses the family, community, and society.

While building psychological resilience can be undertaken from an individual perspective, consideration should also be given to interactions people have with the wider society. Psychological resilience cannot be separated from resilience at the family, community, or societal level as there are clear linkages between these aspects. Indeed, Norris and colleagues (2008) reiterate that a 'collection of resilient individuals does not guarantee a resilient community' (p. 128) and acknowledges the importance of incorporating broader community aspects in resilience-building.

Research in disaster-related disciplines has shown that communities have many inherent qualities that contribute to building resilience both in times of quiescence, during a disaster, and after a disaster. A number of researchers undertaking disaster research consider community resilience as relating to an 'adaptive capacity' held by individuals or communities. This capacity is associated with physical, psychological, social, and economic resources that exist within, or are available to a community (Berkes, 2007; Klein, Nicholls, \& Thomalla, 2003; Mowbray et al., 2007; Norris et al., 2008; Paton, 2006).

In the context of disasters, community resilience should be considered both before and after an event. The period during quiescence before an event occurs is particularly important as this is when much community resilience building can occur, and thus contributes to people's adaptive capacity post-event. Research into the development of resilience prior to a disaster has identified a number of community and societal attributes that contribute to resilience building. These include community participation; sense of community; articulation of problems; social norms, collective efficacy; social support; trust; empowerment, and the availability of physical and emotional resources (Lindell \& Perry, 2000; Lindell \& Whitney, 2000; McIvor \& Paton, 2007; Norris et al., 2008; Paton, 2006; Paton, 2007a, 2007b). While many of these attributes have proved to be important in the development of resilience for disasters, many of these qualities operate in daily life and contribute to the overall wellbeing and strength of a community on a regular basis. Such attributes are thus best developed during times of calm, so that communities are robust from a day-today perspective, and may also adapt during times of adversity.

After an event, people may be presented with issues that are different to those encountered as part of daily life. For example, people may not have direct access to shopping centres to get food after a disaster. In dealing with such issues, people may be able to rely on attributes built during times of quiescence (e.g., use linkages built as part of community participation to access food), but some may need additional assistance or intervention to assist with recovery. Such interventions will help with building resilience for the future. Various authors have suggested community-based approaches for increasing resilience after traumatic events have occurred. Landau and colleagues (2008) suggested an approach termed the Link Intervention Targeting the Community Level (LINC). The LINC approach is focussed on reconnecting traumatised individuals with family and the community with the aim of reconnecting pathways, reuniting communities, and enhancing communities. Other authors (e.g., Gordon, 2004) also emphasise the need to re-connect damaged social pathways in communities after a traumatic event to aid recovery.

Walsh (2007) postulated another example of an integrated approach to treating trauma and building resilience. Walsh suggested that to facilitate healing and resilience post-event, individuals, families and communities should actively engage in four main processes, which include: sharing and acknowledging the reality of a traumatic event and the losses associated with that event; sharing experiences of loss and survivorship; participating in the reorganisation of family and community including planning for survivors well-being; and reinvesting in relationships and life pursuits.

Such examples illustrate that when building resilience, it is important that family, community, and societal linkages are taken into account as part of the wider environment. As resilience has definite links with wider community aspects, we suggest that three key levels of external support should be considered when building psychological resilience: family support, community support and societal support.

Family support. Family support is essential to building psychological resilience. Family members are often trusted members of an individual's support system, and as well as providing direct support to an individual, can also provide linkages back to the wider community (Landau et al., 2008; Walsh, 2007). Family may often feel a greater sense of responsibility for other family members and may be more likely to become involved in 


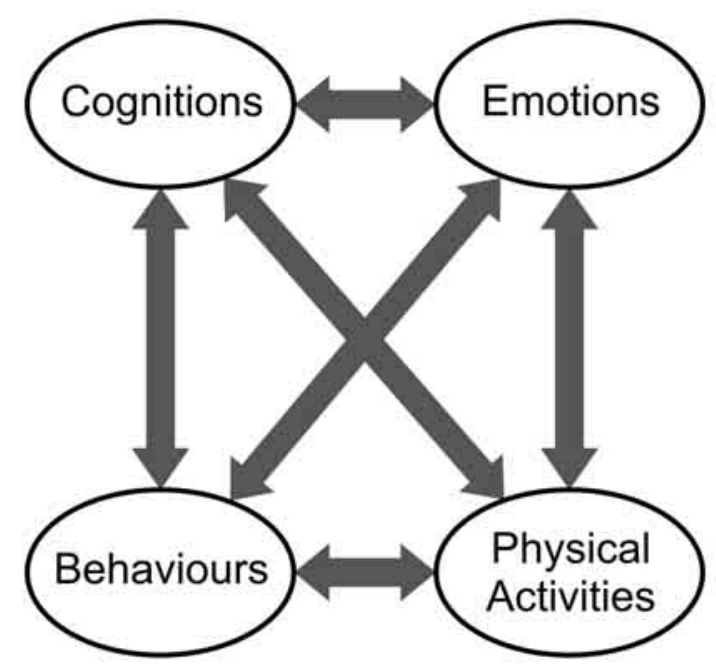

ENVIRONMENT

Figure 1

The Five Part Model.

interactions that will assist in developing resilience or reducing trauma. From a disaster perspective, family members may be more likely to interact, collect, or build resources that will assist with surviving and adapting after a disaster (e.g., collect physical preparedness survival items to protect a spouse or children).

Community support. Communities are often defined as a group of individuals who interact due to their social network and defined territory (Johnston, Gregory, \& Smith, 1994). However, despite the traditional definition, communities do not necessarily have to be defined by being in a specific geographic location, as the term 'community' can also refer to groupings of people who share common interests. The key to a community is that it operates based on a social network whereby people interact with one another in some way. Thus, communities can be neighbourhood based, or based on widespread social networks related to religion, family ties, culture, or social activities. Community support can be harnessed for resilience. In particular, community development programs can assist in developing both individual psychological and community strengths required for resilience (Paton, 2006). For example, community development programs can help build individual attributes such as self-efficacy and problem solving ability, both of which link back to a person's psychological resilience. They also can build strong community attributes such as community participation, sense of community, collective efficacy, and articulation of problems, which can influence people to prepare for a disaster, or provide communities with social and physical resources to cope with the impacts of one (Paton, McClure, \& Burgelt, 2006).
Societal support. Resilience building is also important from a broader societal level, as the availability of societal resources can have an influence on psychological, family and community resilience. For example, having trust in a societal institution (e.g., a health provider, emergency management office, or local authority) will mean that an individual may be more likely to interact with that institution for a positive outcome. A lack of trust in that institution will have the opposite effect (Paton, 2007b). Likewise the availability of physical and social resources (e.g., social support) will have an influence on what individuals, families and communities can practically do to prepare for and respond to the impacts of a disaster (Norris et al., 2008; Paton, 2006).

\section{Integrated Model of Resilience}

Figure 2 presents a new model for understanding and developing psychological resilience. The main structure of the original Five Part Model for psychological resilience remains, with consideration given to cognitions, emotions, behaviours, and physical activities and the interactions between these aspects. We propose that many of the community and societal aspects that make up community resilience fit into the environment section of the Five Part Model. Therefore family, community and societal support should be considered and accounted for as part of the wider environment.

\section{The Future: Where to From Here?}

A central criticism in the area of resilience research has been the lack of resilience models that can be scientifically tested and the lack of construct validation (Masten,

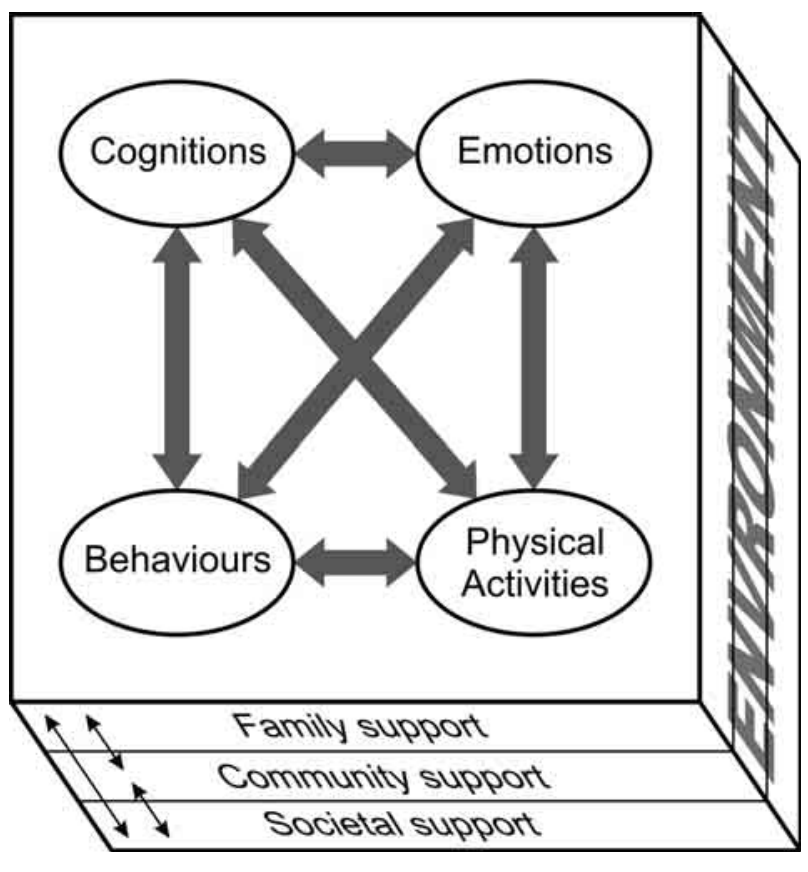

Figure 2

Five Part Resilience Model. 
1999). Therefore, the Five Part Resilience Model needs to be evaluated as a framework for building resilience, both from the psychological viewpoint and the community viewpoint. In addition, this model gives a framework for a multi-disciplinary approach. From a practitioner viewpoint the Five Part Model has shown to have clinical utility (Giarratano, 2004), but the Five Part Model has predominantly been used to understand what has already happened to an individual (e.g., posttraumatic stress disorder). Thus, the Five Part Resilience Model could be used as a framework after the occurrence of adverse events. However, more importantly, this framework has great potential for building individual and community resilience before adverse events through individual programs, community-based intervention programs, and development programs.

\section{References}

Agaibi, C.E., \& Wilson, J.P. (2005). Trauma, PTSD, and resilience: A review of the literature. Trauma, Violence, and Abuse, 6, 195-216.

Beck, J.S. (1995). Cognitive therapy: Basics and beyond. New York: Guilford Press.

Berkes, F. (2007). Understanding uncertainty and reducing vulnerability: Lessons from resilience thinking. Natural Hazards, 41, 283-295.

Bonanno, G.A. (2004). Loss, trauma, and human resilience: Have we underestimated the human capacity to thrive after extremely aversive events? American Psychologist, 59, 20-28.

Buckley, T.C., Mozley, S.L., Bedard, M.A., Dewulf, A.C., \& Greif, J. (2004). Preventive health behaviors, health-risk behaviors, physical morbidity, and health-related role functioning impairment in veterans with post-traumatic stress disorder. Military Medicine, 169, 536-540.

Chang, E.C. (2002). Optimism-pessimism and stress appraisal: Testing a cognitive interactive model of psychological adjustment in adults. Cognitive Therapy and Research, 26, 675-690.

Curran, J., Machin, C., \& Gournay, K. (2006). Cognitive behavioural therapy for patients with anxiety and depression. Nursing Standard, 21, 44-52.

Giarratano, L. (2004). Clinical skills for managing PTSD: Proven practical techniques for treating posttraumatic stress disorder. Sydney, Australia: Talomin Books.

Gordon, R. (2004). The social system as site of disaster impact and resource for recovery. The Australian Journal of Emergency Management, 19, 16-22.

Harvey, J., \& Delfabbro, P.H. . (2004). Psychological resilience in disadvantaged youth: A critical review. Australian Psychologist, 39, 3-13.

Hunt, N., \& Evans, D. (2004). Predicting traumatic stress using emotional intelligence. Behaviour Research and Therapy, 42, 791-798.

Hyman, O. (2004). Perceived social support and secondary traumatic stress symptoms in emergency responders. Journal of Traumatic Stress, 17, 149-156.
Johnston, R.J., Gregory, D., \& Smith, D.M. (Eds.). (1994). The dictionary of human geography (3rd ed.). Oxford, England: Blackwell Publishers.

Klein, R., Nicholls, R., \& Thomalla, F. (2003). Resilience to nautral hazards: How useful is this concept? Environmental Hazards, 5, 35-45.

Kumpfer, K.L. (1999). Factors and processes contributing to resilience: The resilience framework. In M.D. Glantz \& J.L. Johnson (Eds.), Resilience and development: Positive life adaptations (pp. 179-224). New York: Kluwer Academic/Plenum Publishers.

Lahad, M. (1993). The story of coping resources. In M. Lahad \& A. Cohen (Eds.), Community stress prevention (pp. 117-143). Kiriat Shmona, Israel: Community Stress Prevention Centre.

Landau, J., Mittal, M., \& Wieling, E. (2008). Linking human systems: Strengthening individuals, families and communities in the wake of mass trauma. Journal of Marital and Family Therapy, 34, 193-209.

Leahy, R.L., \& Holland, S.J. (2000). Treatment plans and interventions for depression and anxiety disorders. New York: Guilford Press.

Lindell, M.K., \& Perry, R.W. (2000). Household adjustment to earthquake hazard: A review of research. Environment and Behavior, 32, 461-501.

Lindell, M.K., \& Whitney, D.J. (2000). Correlates of household seismic hazard adjustment adoption. Risk Analysis, 20, 13-25.

Masten, A.S. (1999). Resilience comes of age: Reflections on the past and outlook for the next generation of research. In M.D. Glantz \& J.L. Johnson (Eds.), Resilience and development: Positive life adaptations (pp. 281-296). New York: Kluwer Academic/Plenum Publishers.

McIvor, D., \& Paton, D. (2007). Preparing for natural hazards: Normative and attitudinal influences. Disaster Prevention and Management, 16, 79-88.

Mohaupt, S. (2008). Review article: Resilience and social exclusion. Social Policy and Society, 8, 63-71.

Montes-Berges, B., \& Augusto, J.M. (2007). Exploring the relationship between perceived emotional intelligence, coping, social support and mental health in nursing students. Journal of Psychiatric and Mental Health Nursing, 14, 163-171.

Mowbray, C.T., Woolley, M.E., Grogan-Kaylor, A., Gant, L.M., Gilster, M.E., \& Shanks, T.R.W. (2007). Neighborhood research from a spatially oriented strengths perspective. Journal of Community Psychology, 35, 667-680.

Norris, F.H., Stevens, S.P., Pfefferbaum, B., Wyche, K.F., \& Pfefferbaum, R.L. (2008). Community resilience as a metaphor, theory, set of capacities, and strategy for disaster readiness. American Journal of Community Psychology, 41, $127-150$.

Paton, D. (2006). Disaster resilience: Building capacity to coexist with natural hazards and their consequences. In D. Paton \& D. Johnston (Eds.), Disaster resilience: An integrated approach (pp. 3-10). Springfield, IL: Charles C Thomas. 
Paton, D. (2007a). Measuring and monitoring resilience in Auckland. GNS Science Report. (No. 2007/18). Wellington, New Zealand: GNS Science.

Paton, D. (2007b). Preparing for natural hazards: The role of community trust. Disaster Prevention and Management, 16, 370-379.

Paton, D., McClure, J., \& Burgelt, P.T. (2006). Natural hazard resilience: The role of individual and household preparedness. In D. Paton \& D. Johnston (Eds.), Disaster resilience: An integrated approach (pp. 105-124). Springfield, IL: Charles C. Thomas.

Polk, L. V. (1997). Toward a middle-range theory of resilience. Advances in Nursing Science, 19, 1-13.
Rutter, M. (2006). Implications of resilience concepts for scientific understanding. Annals New York Academy of Sciences, 1094, 1-12.

Stephens, C.V. (1997). Debriefing, social support and PTSD in the New Zealand Police: Testing a multidimensional model of organisational trauamtic stress [Electronic Version]. The Australasian Journal of Disaster and Trauma Studies, 1. Retrieved April 24, 2007, from http://www. massey.ac.nz/\%7Etrauma/issues/1997-1/cvs1.htm

Walsh, F. (2007). Traumatic loss and major disasters: Strengthening family and community resilience Family Process, 46, 207-227.

Williams, C., \& Garland, A. (2002). A cognitive-behavioural therapy assessment model for use in everyday clinical practice. Advances in Psychiatric Treatment, 8, 172-179. 